(C) Elsevier Sequoia S.A., Lausanne - Printed in The Netherlands

\title{
WEAR CHARACTERISTICS OF PORCELAIN DENTURE TEETH*
}

\author{
C. N. RAPTIS, J. M. POWERS and P. L. FAN
}

School of Dentistry, The University of Michigan, Ann Arbor, MI 48109 (U.S.A.)

(Received June 2, 1980)

\section{Summary}

Surface wear and frictional behavior of porcelain denture teeth were examined with a single-pass sliding technique. The influence of environment, type of surface (enamel or dentin) and repeated passes on the wear characteristics of the teeth were investigated. The enamel surface of the teeth was more resistant to penetration and to surface damage than the dentin surface was. Damage was more severe for double-pass than for single-pass sliding. The wear characteristics were affected similarly by the liquid environments of water and of human saliva. Quantitative differences in the wear parameters investigated were found between the two brands of porcelain denture teeth.

\section{Introduction}

One approach to the study of wear involves the characterization of surface failure under conditions of single-pass sliding. Such an approach has been used in dentistry to study the wear characteristics of human enamel $[1,2]$, dental amalgam [3], dental feldspathic porcelain [4], dental composite and unfilled resins [5] and dental pit and fissure sealants [6].

The purpose of this study was to characterize the surface failure of porcelain denture teeth and to investigate the influence of environment, type of surface (enamel or dentin) and repeated passes on the wear characteristics of these teeth.

\section{Materials and methods}

Porcelain denture teeth MP (mold 52L, body 120, enamel 207, Myerson Toulh Corp., Cambridge, MA 02139) and DP (mold 11H, body 37, enamel 88, Dentsply International Inc., York, PA 17404) were evaluated. The

*This investigation was presented in part at the annual meeting of the American Association for Dental Research in Los Angeles, CA, March 1980. 
apparatus used has been described in detail elsewhere [7, 8]. It consisted of a surface grinder, loading jig, diamond slider, friction transducer and sample holder. A diamond hemisphere (360 $\mathrm{m}$ in diameter) was slid across the surface of the specimens. The sample holder was mounted on the table of a surface grinder moving horizontally at a speed of $0.025 \mathrm{~cm} \mathrm{~s}^{-1}$. Parallel one-traversal scratches that resulted from sliding a normal load of $0.5-10 \mathrm{~N}$ in varying increments were made on each specimen.

The study was divided into four parts.

(1) The influence of liquid media on the wear characteristics of the enamel as-received surfaces of DP teeth was investigated using distilled water and filtered human saliva.

(2) The wear characteristics of the enamel of MP teeth under single-pass sliding were investigated using distilled water as the liquid medium.

(3) The wear characteristics of the dentin of teeth from both manufacturers under single-pass sliding were investigated in distilled water. The dentin surface was prepared by uniformly removing material from the ridge lap area of the denture teeth to a depth of $1 \mathrm{~mm}$ and subsequent polishing.

(4) The influence of double-pass sliding on the wear characteristics of the enamel as-received surface of teeth from both manufacturers was studied using distilled water as the liquid medium. Two one-traversal scars were superimposed on each other in the same sliding direction with the same normal load.

Five specimens were tested for each material and each condition. Tangential force and track width data were collected for each run with one exception: tangential force results were not obtained for the double-pass runs. Track width was measured with a metallographic microscope and a calibrated eyepiece. Surface failure modes were determined by optical microscopy at a magnification of $200 \times$ and were further studied by scanning electron microscopy (SEM). Wear scars were classified according to the degree of surface damage on a one to five ordinal scale. Class 1 was ductile failure, class 3 was tensile failure characterized by surface tensile cracks and class 5 was catastrophic failure featuring chevron formation. Damages intermediate to those of classes 1 and 3 and classes 3 and 5 were classified as class 2 and class 4 respectively.

The tangential force and track width data were evaluated as a function of normal load by an analysis of variance [9] that included regression and covariance models to study the effects of medium, condition and manufacturer. To analyze the classified failure data the Spearman-Kärber test [10] was used to obtain an estimate of the transition load at which each sample would begin to show failure appropriate to the next higher classification. The transition loads were used as the response data in a multivariate analysis [9]. The multivariate analysis investigated first whether any differences in the vectors of mean transition loads were present and then whether there were differences in the individual mean transition loads using Scheffe's method [11]. A 95\% level of confidence was used for all the statistical analyses performed. 


\section{Results}

The track width, tangential force and classified surface failure results for DP teeth in water were not statistically different from the respective data for runs in saliva, indicating that the wear characteristics of the teeth tested were affected similarly by the two liquid environments. The comparative statistical results of track width and tangential force for water and saliva are presented in Table 1.

Average values of track width for DP and MP teeth are plotted as a function of normal load on log-log coordinates in Fig. 1 and Fig. 2 respectively. The measured values of track width are also compared with values (the straight lines in the figures) computed from an equation derived from a special case of Hertz's [12] theory of contact between two elastic spheres. The equation used was

$$
w=1.82(W R)^{1 / 3}\left\{\frac{E_{\mathrm{B}}\left(1-\nu_{\mathrm{A}}^{2}\right)+E_{\mathrm{A}}\left(1-\nu_{\mathrm{B}}^{2}\right)}{E_{\mathrm{A}} E_{\mathrm{B}}}\right\}^{1 / 3}
$$

where $w$ is the track width, $W$ is the normal load, $R$ is the radius of the diamond hemisphere and $\nu$ and $E$ are the Poisson's ratio and Young's modulus for porcelain (A) and diamond (B) respectively. In this equation Poisson's ratio was 0.30 and Young's modulus was $930 \mathrm{GN} \mathrm{m}^{-2}$ for diamond. Poisson's ratio was 0.24 and Young's modulus was $82.8 \mathrm{GN} \mathrm{m}^{-2}$ for porcelain. Average values of the tangential force for single-pass sliding of the enamel surfaces of both brands are plotted as a function of normal load in Fig. 3. The tangential
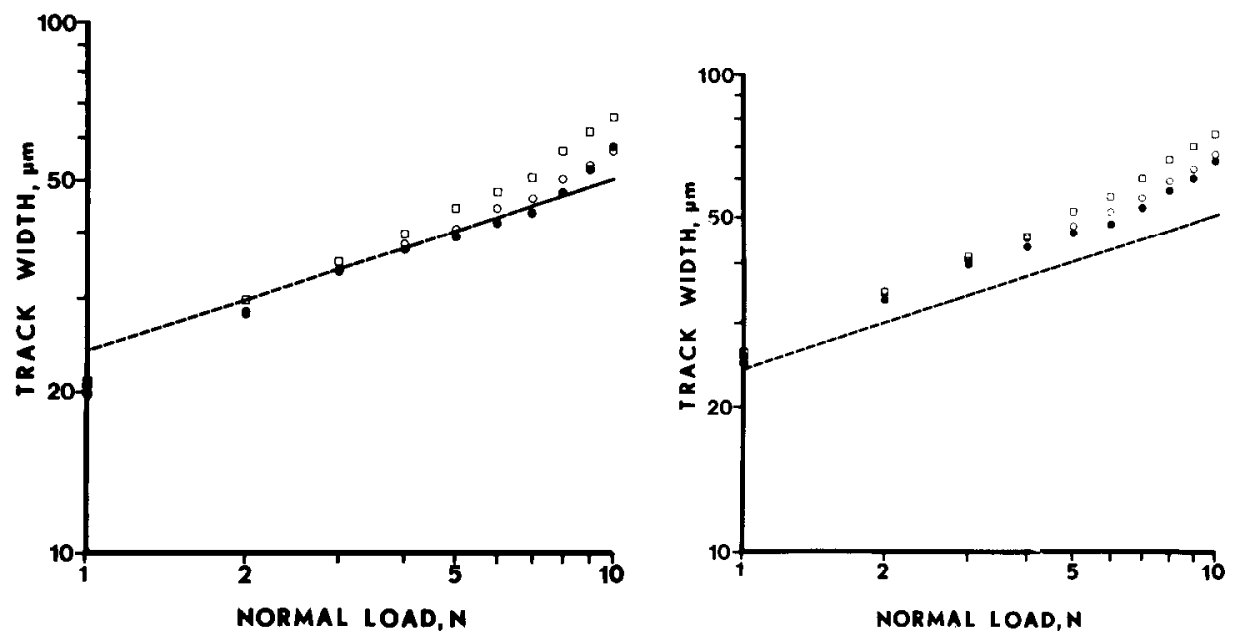

Fig. 1. Log-log plot of track width is. normal load for single-pass sliding on enamel and dentin surfaces and double-pass sliding on enamel for DP teeth: $\bullet$, enamel; $O$, dentin; $\square$, double pass.

Fig. 2. Log-log plot of track width vs. normal load for single-pass sliding on enamel and dentin surfaces and double-pass sliding on enamel for MP teeth: $\bullet$, enamel; $\bigcirc$, dentin; $\square$, double pass. 


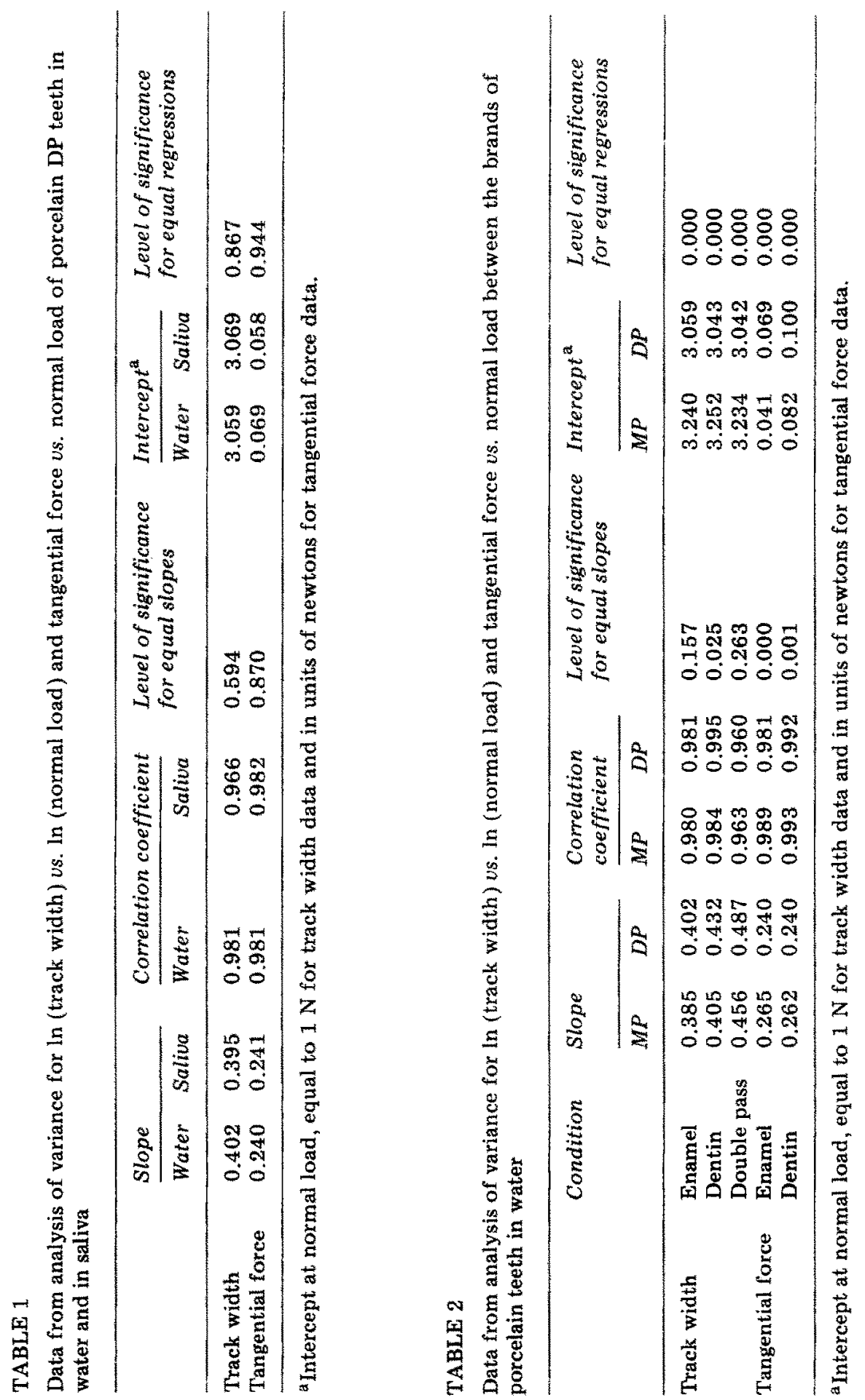




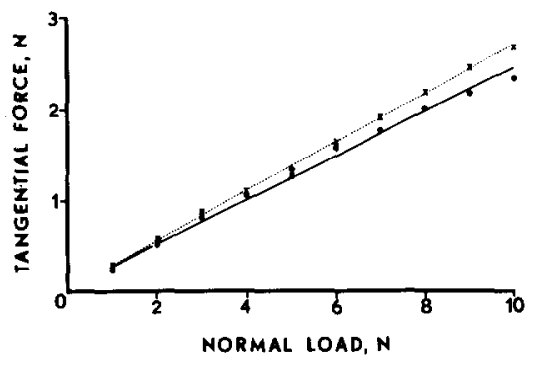

Fig. 3. Tangential force $v$ s. normal load for single-pass sliding of the enamel surfaces of porcelain denture teeth : $x, \cdots--, M P ; \bullet,-, D P$.

force values for the dentin surfaces of the two brands exhibited the same ranking. The comparative statistical results of track width and tangential force data for the various surfaces and treatments are presented in Table 2.

The estimated loads for surface failure of both brands of porcelain teeth are presented in Table 3. Photomicrographs typifying the surface failure classification for both brands of porcelain teeth are shown in Fig. 4. A picture of the ductile mode of failure (class 1 ) was omitted. The modes of surface failure resulting from the second pass are shown in Fig. 5. The increase in the extent of surface damage of the section of the wear tracks corresponding to the second pass is evident, particularly at the larger normal loads.

\section{Discussion}

Porcelain fractured like a brittle substance during sliding. The severity of surface failure varied according to the applied normal load. Low values of tangential force and low values of track width were observed with less severe surface deformation and were associated with the lower values of the range of normal loads applied. High tangential force and track width values were observed with more extensive surface damage and larger normal loads.

\section{TABLE 3}

Estimated loads for surface failure transitions for porcelain teeth (MP and DP)

\begin{tabular}{|c|c|c|c|c|c|c|c|c|}
\hline \multirow[t]{3}{*}{ Condition and test } & \multicolumn{8}{|c|}{ Estimated transition load ${ }^{\mathrm{a}}(\mathrm{N})$} \\
\hline & \multicolumn{2}{|c|}{ Classes 1 - 2} & \multicolumn{2}{|c|}{ Classes $2-3$} & \multicolumn{2}{|c|}{ Classes $3-4$} & \multicolumn{2}{|c|}{ Classes $4-5$} \\
\hline & $M P$ & $D P$ & MP & $D P$ & $M P$ & $D P$ & MP & $D P$ \\
\hline Enamel single pass & 2.45 & 2.65 & 3.95 & 4.55 & 6.45 & 8.20 & - & - \\
\hline Dentin single pass & 2.10 & 2.50 & 3.50 & 4.50 & 6.10 & 6.70 & 9.9 & - \\
\hline Enamel double pass & 1.50 & 2.10 & 3.10 & 4.10 & 4.90 & 5.90 & 7.10 & 8.10 \\
\hline
\end{tabular}

averall statistical test: $F=102.15, p=0.000$ for enamel single pass; $F=4.31, p=0.070$ for dentin single pass; $F=6.86, p=0.029$ for enamel double pass. 


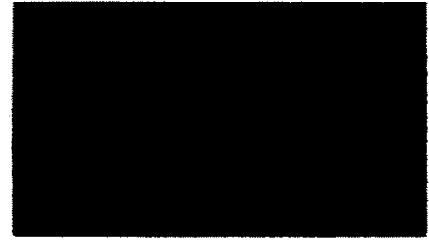

(a)

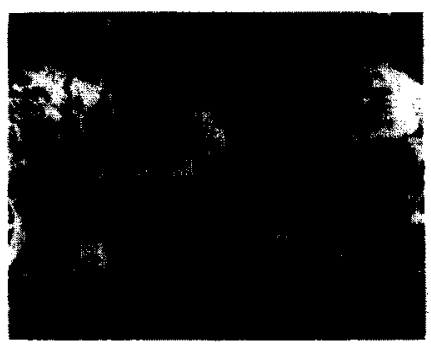

(c)

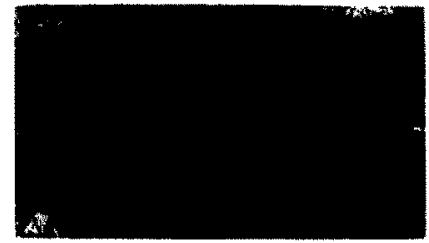

(b)

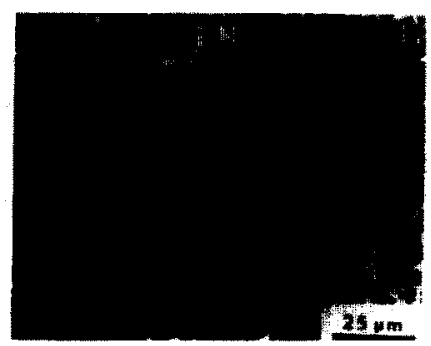

(d)

Fig. 4. SEM photomicrographs of wear tracks showing modes of surface failure of enamel and dentin surfaces of porcelain denture teeth : (a) class 2 ; (b) class 3 ; (c) class 4 ; (d) class 5 . .

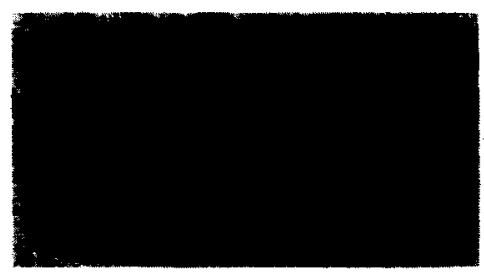

(a)

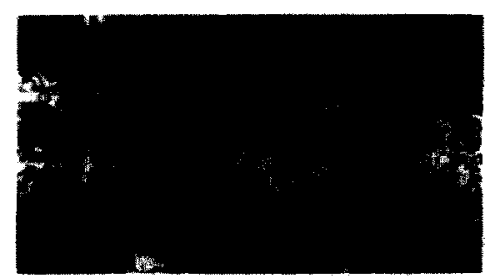

(c)

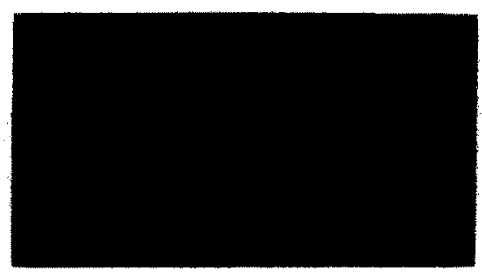

(b)

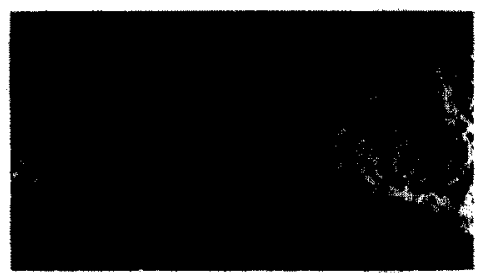

(d)

Fig. 5. Modes of surface failure of enamel surfaces of porcelain denture teeth on changing from single- to double-pass sliding: (a) class $1-2$; (b) class $2-3$; (c) class $3-4$; (d) class $4-5$. 
Three major modes of surface failure were seen over the range of normal loads tested. A ductile mode of failure characterized by smooth grooves was observed at low loads; tensile failure was absent and plastic flow predominated. A ductile-to-brittle transition was observed within a small range of normal loads. These initial load values were different for the various dentures and conditions. This observation suggested that a strain corresponding to these critical normal loads could be accommodated by plastic deformation. Above this strain level, tensile cracks occurred immediately on passing of the slider. The tensile failure producing the cracks appeared to be the combined effect of a hertzian stress (caused by the normally loaded slider) and a stress caused by the frictional forces of sliding. At even larger normal loads a chipping mode of surface failure was observed and the scars no longer showed evidence of ductility. Tensile cracking and chevron formation at the surface adjacent to the wear track were characteristic of extensive surface failure.

The variables of track width, tangential force and surface failure classification were effective in ranking data obtained under single-pass sliding for the two brands of porcelain denture teeth and for the two different surfaces. As a group the enamel surfaces of both brands were less susceptible to surface damage, were more resistant to penetration and produced lower values of frictional force than the dentin surfaces did.

The normal load on enamel at which catastrophic failure occurred at the porcelain surface was larger than the corresponding load on dentin for both MP and DP teeth. The track width values measured for MP teeth were higher than the values predicted by the hertzian analysis of two elastic bodies. The discrepancy was greater at the larger normal loads. It is suggested that these high values of track width reflect the propagation of cracks in the material which is not taken into account in the hertzian model. The material behaved in a more brittle fashion at the larger loads; hence the discrepancy is greater between measured and predicted values at these loads. The track width values for DP teeth were in reasonable agreement with the predicted hertzian values with the exception of those corresponding to the highest loads (9 and $10 \mathrm{~N}$ ). Disturbance of the porcelain surface during the removal of material and the subsequent polishing could be a factor causing the differences between enamel and dentin. Other possible contributing factors could be a difference in composition between the enamel surface and the dentin surface and a difference resulting from the firing technique during manufacturing. The clinical implications of these findings are important since it is common practice to grind the denture teeth in an effort to produce a harmonious occlusal scheme.

Wear caused by double-pass sliding was more severe than might have been expected on the basis of single-pass sliding. When the estimated transition loads corresponding to single- and double-pass sliding were compared for both brands it was observed that the differences in load increased as the severity of failure increased. For both brands track widths in the double-pass regions were higher under the same normal load than those in single-pass 
regions, especially at higher loads. These observations suggest that there is a limit to the amount of plastic deformation that can be undergone during sliding. If this limit were exceeded during a single pass, a subsequent pass would necessarily cause cracking independently of the load of the later pass. Double-pass sliding provides additional information about the nature of subsurface damage that may not be apparent from single-pass tests. In practical sliding systems where repeated sliding is always occurring, wear of brittle materials is probably dominated by the catastrophic mechanisms found on the second pass rather than by the events that occurred during the first pass.

The wear-testing technique for porcelain denture teeth, characterized by single-pass and double-pass sliding, enabled the determination of differences in the wear parameters of various experimental combinations. The enamel of porcelain DP teeth had lower values of track width and tangential force and higher values of estimated loads for surface failure transitions than the enamel of porcelain MP teeth had. On comparison of the three wear parameters among the dentin groups of the two manufacturers the same differences were found. The dentin surface of MP teeth was more susceptible to penetration and surface damage than the dentin surface of DP teeth, while the force required to cause deformation of the dentin of MP teeth was greater than that required for DP teeth. When double-pass sliding was employed on the enamel the ranking between the two brands was consistent with the ranking of the enamel and dentin produced by single-pass sliding.

In a concurrent study [13] acrylic denture teeth from the same two manufacturers were tested under single- and double-pass sliding techniques and their wear characteristics were evaluated. The differences observed between the two materials were characteristic of the essentially brittle nature of porcelain and the ductile nature of acrylic denture tooth materials. The three parameters (track width, tangential force and mode of surface failure) differed between the porcelain and acrylic denture tooth materials in the following ways:(1) for acrylic teeth the track widths measured were larger and showed greater deviations from the values calculated from the hertzian model; (2) the values of tangential force observed for acrylic teeth were higher than the values for the porcelain teeth; (3) the surface failure observed for acrylic teeth was usually ductile. When at the larger loads cracking was observed with acrylic teeth it was almost always confined within the edges of the wear tracks. Brittle failure was observed at lower loads for porcelain tooth surfaces, featuring the characteristic tensile cracks on the sides of the wear tracks.

\section{Conclusions}

The frictional behavior and surface failure of porcelain denture teeth under sliding were investigated to study the influence of environment, type of surface and repeated passes on the wear characteristics. The response of porcelain to wear testing was characteristic of a brittle material. The wear 
characteristics as determined by single-pass sliding were affected similarly by environments of water and saliva. The enamel as-received surfaces of the teeth were less susceptible to surface damage and surface penetration and generated less frictional resistance than the dentin surfaces under singlepass sliding. The damage resulting from double-pass sliding on the enamel surfaces was an accelerative rather than an additive phenomenon. As a group the enamel and dentin surfaces of DP teeth suffered less extensive damage in single-pass sliding than the surfaces of MP teeth did. Double-pass results on the enamel surfaces of the two brands were consistent with this ranking.

\section{Acknowledgments}

This investigation was supported in part by Research Grant DE-03416 and by Service Award DE-07057 from the National Institute of Dental Research, National Institutes of Health, Bethesda, MD 20205.

The cooperation of Dentsply International, Inc., and Myerson Tooth Corporation in providing commercial products is acknowledged.

\section{References}

1 J. M. Powers, R. G. Craig and K. C. Ludema, Frictional behavior and surface failure of human enamel, J. Dent. Res., 52 (1973) 1327.

2 J. M. Powers, R. G. Craig and K. C. Ludema, Wear of dental enamel, Wear, 23 (1973) 141.

3 J. C. Roberts, J. M. Powers and R. G. Craig, Wear of dental amalgam, J. Biomed. Mater. Res., 11 (1977) 513.

4 G. R. Miller, J. M. Powers and K. C. Ludema, Frictional behavior and surface failure of dental feldspathic porcelain, Wear, 31 (1975) 307.

5 J. M. Powers, J. C. Roberts and R. G. Craig, Wear of filled and unfilled dental restorative resins, Wear, 39 (1976) 117.

6 J. C. Roberts, J. M. Powers and R. G. Craig, Wear of commercial pit and fissure sealants, J. Dent. Res., 56 (1977) 692.

7 J. M. Powers and R. G. Craig, Wear of fluorapatite single crystals: I - A method for quantitative evaluation of wear, J. Dent. Res., 51 (1972) 168.

8 J. M. Powers and R. G. Craig, Wear of fluorapatite single crystals: II - Frictional behavior, J. Dent. Res., 51 (1972) 605.

9 A Manual of Elementary Statistics using MIDAS, Statistical Research Laboratory, University of Michigan, Ann Arbor, MI 48109, 1975.

10 D. J. Finney, Statistical Method in Biological Assay, Hafner, New York, 2nd edn., 1964.

11 W. C. Guenther (ed.), Analysis of Variance, Prentice-Hall, Englewood Cliffs, NJ, 1964.

12 H. R. Hertz, On the contact of elastic solids, J. Reine Angew Math., 92 (1881) 156 (in German).

13 C. N. Raptis, Wear characteristics of prosthetic denture teeth, Master's Thesis, School of Dentistry, University of Michigan, Ann Arbor, MI 48109, 1980. 